\title{
LIMB DARKENING OSCILLATIONS : THE DISCREPANCY \\ WITH DOPPLER DATA AND THE 160-MINUTE PERIOD
}

\author{
Raymond YERLE \\ Observatoire du Pic-du-Midi et de Toulouse \\ F-31400 Toulouse France
}

\begin{abstract}
The amplitude discrepancy between solar limb darkening and Doppler measurements in the five-minute range, suggests the possible existence of Earth's atmosphere oscillations. Recent aeronomical observations add strong evidence to this point of view. A possible explanation of the mysterious 160 -min period is presented as a direct consequence. Short period oscillations are another one. Slight discrepancies between Doppler data and between those with theoretical calculations could be also explained in the same way.
\end{abstract}

\section{WHAT KIND OF DISCREPANCY ?}

The discrepancies between solar limb darkening and Doppler measurements are in amplitude for long periods (Fossat et al. 1977; Christensen-Dalsgaard, 1982) and the reason of this discrepancy has yet to be found in spite of several explanations (Hill et al. 1978; Hill and Logan1984) frequency in the five-minute range (Yerle, 1984) and more precisely on rotational splitting in the 5 -min region as well as at longer periods and, as for amplitude, the reason has yet to be found (ChristensenDalsgaard, 1985).

WHY ?

The apparent limb darkening profile $A$ of the Sun is linked to the intrinsic solar one $S$ by the relation :

$$
A=S * B
$$

$B$ being the spread function of the Earth's atmosphere, neglecting the instrumental one. Is the B function purely stochastic? NO ! Stratospheric continuous vertical velocity wind data, obtained from three vertically-directed ST (Stratosphere-Troposphere) radars installed in a closely spaced configuration (4-6 km on a side), indicate on power spectra a well defined spiked structure in the five-minute range, superimposed to a turbulent general shape. The authors of Alpex (Alpine Experiment) remark that this structure is linked to Brunt-Väisälä periods of the Earth's atmosphere (Balsley et al. 1983).

See figures 1 and 2 . 
THE BRUNT-VAISALA PERIODS OF THE EARTH'S ATMOSPHERE

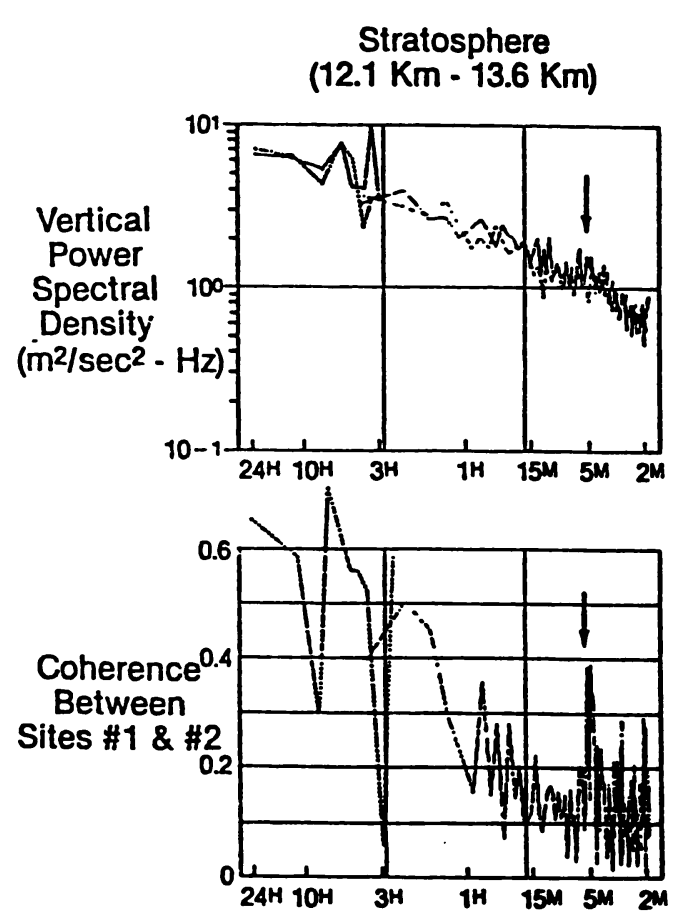

Fig. 1 - In spite of the location of the sites selected to be out of orographic effects and quietest windy conditions, the power and above all the coherence spectra show a strong bump ( 0.29 corresponding to the $3 \sigma^{2}$ confidence level) in the five-minute range (By courtesy of Pr. Crochet).

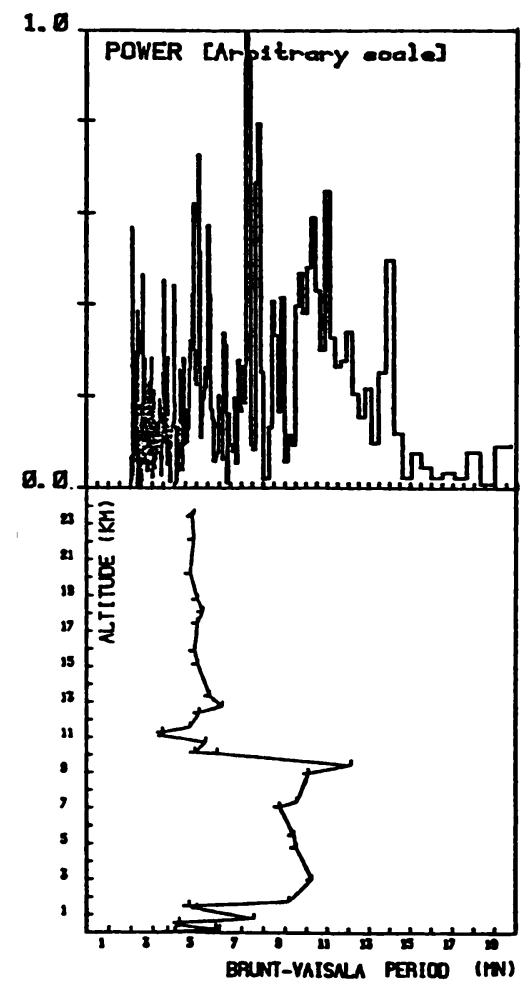

Comparison of an apparent solar limb darkening oscillations power spectrum (above) with Brunt-väisälä terrestrial atmospheric periods computed from radiosonde data (By courtesy of $\mathrm{Dr}$. Klaus).

\section{CONSEQUENCES FOR HELIOSEISMOLOGY ?}

Limb darkening data - The above remarks quite well explain the multifarious discrepancies between limb darkening and Doppler data : we do not observe the same phenomenon by scanning the apparent solar limb and by Doppler measuring the whole Sun. However, from recent theoretical ampli tude calculations (Schmieder and Mein, 1986), we cannot exclude the pre sence of 5-min high degree $\mathrm{p}$-modes.

Doppler data - The telluric atmospheric abundances of atoms used by Dop pler techniques are very faint, so that we can expect little seeing effects on ground-based Doppler data. However, slight differences appear between different sets of Doppler results and between those with space ACRIM data (Woodard, 1984). The Brunt-Väisälä periods, through blending effects of water and oxygen telluric lines, also could be in this case the primary cause (see the results obtained by Kobanov, 1985).

THE 160-MINUTE PERIOD

Maybe a beat phenomenon. See : Yerle,R. 1986, Astron. Astrophys.161,L5 